\title{
Fungal infection in a tertiary care level III NICU: A Review
}

\author{
Authors \\ Dr Kush D Jhunjhunwala ${ }^{1}$, Dr Gurmeet Singh Sarla ${ }^{2}$ \\ ${ }^{1}$ Associate Professor of Paediatrics, Indira Gandhi, Government Medical College, Nagpur 440002 \\ (Maharashtra) Nagpur University \\ ${ }^{2}$ Senior Advisor Surgery, Military Hospital Devlali, Devlali Cantt, Nasik, Maharashtra. Pin 422401
}

\section{Introduction}

Candida infections are an increasing cause of septicemia in neonatal ICUs. Approximately $20 \%$ of very-low-birth-weight (VLBW) (birth weight $<1,500 \mathrm{~g})$ preterm infants experience a serious systemic infection during their initial hospital stay (a). Preterm infants are predisposing to the Candida infections because of immaturity of their immune system and invasive interventions.

Candida species are the $3^{\text {rd }}$ most frequent organism (after coagulase negative Staph. and Staph. Aureus) isolated in the late- onset sepsis in very low birth weight infants(I.e. $<1500 \mathrm{~g}$ ) The rate of fungal sepsis is lower in full-term infants; however the overall morbidity and mortality from disseminated candidiasis are very high, often approaching $25 \%$ to $54 \%$ respectively.

Neonates may acquire Candida by vertical or nosocomial transmission. In vertical transmission, acquisition may occur either during gestation or at the time of delivery. In both cases an ascending route from the mother's vagina is involved. Vaginal candidiasis occurs frequently among pregnant women, especially in the last trimester. Fungal infection in newborn is also an important cause of nosocomial infection in NICU, the incidence being $2.8-12.9 \%$. However, there is an 11 fold increase in the incidence of the same in the last decade.

The early NICU course favors colonization and proliferation of fungi since many VLBW infants have central vascular catheters and are exposed to broad-spectrum antibiotics and parenteral nutrition. The vast majority of fungal infections in preterm neonates are due to Candida species (Commensal yeast, C.albicans, C.tropicalis, C.glabrata), with a small number being due to Malassezia and other rare fungi like Aspergillus's sp etc. Acute mortality from Candida infection may be associated with septic shock due to the production of endotoxin-like substances, hemolysin, and pyrogens by the organism

\section{Fungal infection: Kalawati Experience}

The following is our own experience in level III tertiary care NICU at Kalawati Saran Children Hospital, New Delhi. It's a 400 bedded exclusive children hospital with all the pediatric subspecialty clinics under one roof, and is attached to Lady Hardinge Medical College. The hospital has 35 bedded NICU with 6 ventilatory beds. Case records with clinical details of all the 
neonates admitted to the NICU at the Kalawati Saran Children Hospital for three consecutive years were analyzed for the occurrence of systemic candidiasis. The records were evaluated for gestation age, sex, birth weight, days on antibiotic, mechanical ventilation, peripheral catheterization, paranteral nutrition, and clinical features, bacterial isolated in the candida positive cases, treatment details, and final outcome. Clinically documented fungal infection (presumed fungal infection) was taken as clinical worsening with septic features confirmed by laboratory data (increase in serum C-reactive protein levels \{Positive titers\}), with leucocytosis $\{>30000 / \mathrm{mm} 3\}$ or leucopenia $\{<6000 / \mathrm{mm} 3\}$ and/or thrombocytopenia $\{<50000 / \mathrm{mm} 3)$ despite aggressive broad spectrum antibiotic therapy for at least 2 days, with no isolation of other microorganisms in any cultures in the last 2 days. Diagnosis of the systemic candidiasis was made if candida was isolated from one or more normally sterile body fluids (Blood, CSF/urine). The day of onset of systemic candidiasis was defined as the day of life on which Candida was first isolated from a normally sterile site. Resistance to the antifungal agent was defined as absence of clinical response or deterioration (other causes being ruled out) and /or body fluid culture or urine specimen continuing to grow candida beyond seven days of treatment.

Out of the total of 987 admissions in the NICU in the said period, 47 neonates acquired systemic candidiasis. The mean age of the onset of systemic candidiasis was 12.8 days, the range being 6-19 days.

Various risk factors associated with candidiasis and there frequencies are shown in the Table 1.

All the babies had a history of peripheral catheterization and had received broad spectrum antibiotics. $91.4 \%$ were premature and $80 \%$ were low birth weight. The mean gestational age was 31 wks (28-37) and the mean birth weight was 1040gms (760-3100).About 18 babies (38.29\%) required mechanical ventilation support and 26 babies $(55.31 \%)$ had CVC.
The clinical feature was predominantly related to the respiratory system (persistent/recurrent pneumonia, recurrent apnea) and gastrointestinal tract (high gastric aspirate, abdominal distention), though non specific features like lethargy were also common Table 2 . In our study we also notice that generalized edema and an ashen grey color of the neonate also gives an important clue to the associated fungal infection.

Associated bacteraemia was present in $80 \%$ of the babies and mean number of days on antibiotic was 19.8 (13-24). The bacteria isolated included Klebsiella pneumonias, Pseudomonas aeruginosa, Enterobacter aerogenes and Acinetobacter anitratus. Blood and urine was the common site for the fungal growth. Thrombocytopenia was found in all (100\%) of the babies with fungal infection and the mean WBC count was 7340(4000-14000). Amphotericine B was the antifungal drug used to treat the babies with systemic fungal infection and fluconazole was used as an add on therapy in only 4 (8\%) babies who were not responding to the standard line of treatment. Of the complications, hypokalemia was seen in $2(4.25 \%)$ babies, $>25 \%$ increase in $\mathrm{Sr}$. Creatinine in only $1(2.12 \%)$, and hypoalbuminemia in $10(21.27 \%)$. There were total 8 deaths and we were not sure wheather the neonates expired primarily due to fungal or bacterial infection but associated causes which contributed to there deaths were present like intracranial haemorrage, bacterial sepsis, meningitis, severe birth asphyxia with multiple organ dysfunction, and massive pulmonary haemorrhage. 
Table 1 Risk Factors Associated With Candidiasis

Risk Factor

Prematurity

$\mathrm{n}$ (frequency)

Low birth weight

43

37

Ventilated Babies

18

47

47

09

Parenteral Nutrition

CVC
Percentage

91.4

80.0

38.29

100

100

19.10

55.31

Table 2 Clinical features in neonates with candidiasis

$\begin{array}{lcc}\text { Respiratory symptoms } & 28 & 59.57 \\ \text { Recurrent apneoa } & 11 & 23.40 \\ \text { Lethargy } & 23 & 48.93 \\ \text { Increased gastric aspirate } & 19 & 40.42 \\ \text { Abdominal distention } & 15 & 31.91 \\ \text { Edema } & 11 & 23.30 \\ \text { Ashen Grey Colour } & 07 & 14.89 \\ \text { Hepato-spenomegaly } & 03 & 06.38 \\ \text { Bilateral Renal enlargement } & 02 & 04.20\end{array}$

\section{Discussion}

Candida species are a common cause of neonatal nosocomial bloodstream infections in premature infants and are a leading cause of infectiousrelated mortality in the neonatal intensive care unit (NICU $)^{[1]}$ Systemic candidiasis is increasingly recognized as a major problem in present day NICU. Onset of candidiasis was much earlier in the current study (mean age 12.8days) compared to previous studies in which the mean age of onset ranged from 17-36 days ${ }^{(2)}$. This earlier onset may be due to increased incidence of prematurity in our study group, increased use of broad spectrum antibiotics in our unit and multiple interventions required during the treatment of such babies.

Weese Mayer et $\mathrm{al}^{(3)}$, in their case control study, found the duration of antibiotic therapy as the most significant risk factor associated with candidemia by discriminate analysis. Similar were the findings of Kleigman et $\mathrm{al}^{(4)}$ and Findland el $\mathrm{al}^{(5)}$. Broad spectrum antibiotics use suppresses the normal bacterial flora thereby allowing candida to flourish. Also, an inadequate

anatomical barrier, immune defects, and the presence of the peripheral catheter makes the neonates prime target for fungal invasion. Thus, broad-spectrum antibiotics (e.g., third-generation cephalosporins) enhance fungal colonization by destroying competing bacterial flora ${ }^{[6]}$

A second predisposing factor for the fungal infection in neonates is the use of indwelling central venous or arterial catheters. These provide a portal of entry through an intact cutaneous barrier. $^{(7)}$ In our case, 26(55.31\%) babies with fungal infection had CVC in place. Similar were the findings of Moise $\mathrm{A}$ at el in their studies on colonization and infection of umbilical catheters in newborn infants. Similarly prolonged endotracheal intubation ( $38.2 \%$ in our case) and prolonged parenteral nutrition also predisposes the neonates to acquire fungal infection. Similar were the findings of Weese-Mayer el $\mathrm{al}^{(3)}$ were they showed that the duration of administration of hyperalimentation fluids and of fat emulsions were related to increase risk of fungal infections in premature neonates. ${ }^{(8)}$ 
Ninety one percent of our cases were premature and eighty percent had low birth weight. Other studies have found the incidence of prematurity and low birth weight to range from zero to 35 percent. ${ }^{(9)}$

The predominant clinical manifestation in our study was related to the respiratory system involvement. Others like abdominal distention, high gastric aspirates, lethargy etc are not specific for Candida infections. Therefore, a high degree of suspicion and prompt investigation is mandatory especially in those babies who are premature and present with these symptoms while on broad spectrum antibiotics. The classical clinical picture of systemic candidiasis in neonate is indistinguishable from bacterial sepsis. Hence, clinically fungal infection should be suspected in all the premature neonates specially those with VLBW who's NICU stay has been more than 7 days, has received higher antibiotics for sufficient period of time but is still showing no improvement Consider Candida in the differential of neonatal sepsis, particularly late-onset sepsis. When blood culture is positive for Candida, a through evaluation to rule out disseminated infection should include cultures of urine and CSF, ophthalmological examination, echocardiogram, renal ultrasound and, if clinical signs of arthritis or osteomyelitis are present, radiographic skeletal survey and consider diagnostic aspiration of the affected area.

Blood was the chief site of occurrence of Candida but it takes several days to diagnose fungal infection. Still it remains the Gold standard for the diagnosis of systemic fungal infection in newborns. Candida grows well on routine media. However, it should be taken with strict aseptic precaution and from a peripheral vein only and if the initially $24 / 48 \mathrm{hrs}$ report is negative, then we should wait till 5 days for the candida to grow.

We examines supra-pubic urine for the budding yeast cells to enable an early diagnosis and thereby initiate antifungal therapy pending blood and urine culture reports wherever possible.
Whenever neurological involvement is suspected, Lumber puncture is a must, both for the diagnosis as well as to decide the course of the therapy. Newer rapid diagnostic methods under investigation are PCR, Antigen detection and Molecular diagnostic probes.

Culture proven bacterial sepsis was present in eighty percent of the cases at the onset of candidiasis. Similarly, Faix ${ }^{(10)}$ reported 33 percent of cases to have co existent bacterial sepsis at onset of candidiasis. Although the benefits of empirical therapy in premature infants have not been evaluated in the context of a well-powered randomized trial, there are several small studies that suggest benefit. In a retrospective study of infants admitted to the NICU, empirical antifungal therapy in infants with candidemia was associated with decreased incidence of disseminated infection and reduced mortality ${ }^{(11)}$. This study suggested amphotericin B deoxycholate as a reasonable choice for empirical therapy in an infant with birth weight $<1500 \mathrm{~g}$ who is deteriorating despite usual empirical antibacterial treatment and who has 6 of the following 9 clinical features: admission to an intensive care unit with a substantial rate of candidemia; significant history of broad-spectrum antibiotic coverage; administration of a third-generation cephalosporin; negative blood culture result; falling platelet count; exposure to systemic steroids; not currently being fed; intubated; and cardiovascular instability.

The management consists of giving a good supportive care, maintaining proper temperature, fluid and electrolyte balance, proper nutrition support and treatment of coexistent bacterial infection if any. Strategies for the prevention of neonatal fungal infections have been recently reviewed.

The specific treatment consists of giving antifungal agents. Amphotericin B alone or in combination with 5-fluorocytosine remains the standard of care for neonatal candidiasis. The optimal duration of therapy is unknown. However, it is recommended to complete a minimun of 10 to 
$15 \mathrm{mg} / \mathrm{kg}$ of amphotericin $\mathrm{B}^{(12)}$ in cases of uncomplicated catheter-related candidemia and between 25 to $30 \mathrm{mg} / \mathrm{kg}$ total for patients with invasive disease $^{(13)}$.

Amphotericin B is the drug of choice, and is the most potent antifungal agent available. It is isolated from streptomyces nodosus. It exerts its mechanism of action and toxicity through binding to ergosterol in the cell membrane of fungal and host cells, resulting in formation of membrane pores, cell depolarization followed by cell death It is available in a powder form. A $50 \mathrm{mg}$ vial is reconstituted in $5 \mathrm{D}$ and it is given as an infusion over 4-6 hours. Half life of the drug is about 15 days. Side effects include nephrotoxicity, hypokelemia hypomagnesemia, anaemia, thrombocytopenia, and infusion reaction (temperature and homodynamic instability.) Hence, during therapy with amphotericin, renal functions, hepatic functions, serum electrolyte and blood counts have to be monitor to reduce the chances of side effects.

Newer preparations of Amphotericine are available in the market like AMB Colloidal dispersion, AmBiSome-Liposomal AMB and ABLC-AMB lipid complexs. The advantages are less toxicity, duration of therapy is less (\#2 wks) and it is equal or more effective than the conventional therapy.

Fluconazole, a fungistatic drug, is the most effective of the azoles. Hepatotoxicity, the main side effect, is transient and resolves with cessation of therapy. Fluconazole is a potent inhibitor of the fungal cytochrome p450 and sterol C-14 demethylation. It alters cell membranes, resulting in increased membrane permeability and impairment of purine and pyrimidine precusor uptake for DNA synthesis. Fluconazole is an excellent drug for prophylaxis due to its long halflife, high CSF (70 to 90\%) and pulmonary (120\%) penetration, low lipophilicity, and low protein binding. In one of the biggest study on the use of prophylactic fluconazole in preventing fungal colonization and fungal systemic infections in preterm neonates, ${ }^{(14)}$ Paolo $\mathrm{M} \mathrm{el}^{(15)}$ concluded that prophylactic fluconazole significantly reduces the incidence of both in ELBW and as well as in VLBW babies and decreases the rates of progression from initial colonization to massive colonization and to systemic infection.

Flucytosine (5-FC) interfere with the DNA synthesis. Because of the toxicity and development of the resistant strains, it is of limited use in neonatal infections. However if the infant can tolerate oral medications, flucytosine is very useful for CNS infections and any act synergistically with amphotericin B.

Disseminated candidiasis may be prevented by following some of the following measures

- Hand washing.

- Strict asepsis during invasive procedures.

- Rational use of the antibiotics.

- Asepsis while preparing formula feeds, infusion lines and TPN solutions.

- Prophylactic fluconazole therapy (Risk of azole resistant strains).

\section{Conclusion}

Fungal sepsis remains one of the most important causes of high morbidity and mortality in the neonatal intensive care units. Early recognition and prompt treatment would go a long way in decreasing the severe systemic and disseminated complications occurring due to this disorder. The key message from this article is to consider fungal sepsis in any sick neonate with an extended neonatal ICU stay who has persistent signs of sepsis despite prolonged broad spectrum antibiotic course.

\section{References}

1. Benjamin DK, Jr, DeLong ER, Cotton CM, Garges HP, Steiback WJ, Clark RH. Mortality following blood culture in premature infants: increased with gramnegative bactermia and candidemia, but not gram-positive bacteremia. J Perinatol. 2004; 24:175-180. [PubMed] [Google Scholar] 
2. Clinical microbiology of bacterial and fungal sepsis in very-low-birth-weight infants. Kaufman D, Fairchild KD Clin Microbiol Rev. 2004 Jul; 17(3):638-80, table of contents.

3. Weese-Mayer DE, Fondriest DW, Brouillette RT, Shulman ST. Risk factors associated with candidemia in the neonatal intensive care unit: a case-control study. Pediatr Infect Dis J 1987; 6: 190-196.

4. Kleigman, R.M., Boxerbaum,B., Fanaroff, A.A. 1986. Fungalcolonization in the very low birthweight infant. Pediatrics, 78: 22532

5. Findland, M. and M. W. Barnes (1977). "Changes in Occurrence of Capsular Serotypes of Streptococcus pneumoniae at Boston City Hospital During Selected Years between 1935 and 1974." The Journal of Clinical Microbiology 5: 154166.

6. Clinical microbiology of bacterial and fungal sepsis in very-low-birth-weight infants. Kaufman D, Fairchild KD Clin Microbiol Rev. 2004 Jul; 17(3):638-80,

7. Strategies for prevention of neonatal invasive candidiasis. Kaufman DSemin Perinatol. 2003 Oct; 27(5):414-24

8. Systemic candidiasis in very low-birthweight infants (less than 1,500 grams). Johnson DE, Thompson TR, Green TP, Ferrieri P Pediatrics. 1984 Feb; 73(2):138-43.

9. Neonatal candidiasis among extremely low birth weight infants: risk factors, mortality rates, and neurodevelopmental outcomes at 18 to 22 months. Benjamin DK Jr, Stoll BJ, Fanaroff AA, McDonald SA, Oh W, Higgins RD, Duara S, Poole K, Laptook A, Goldberg R, National Institute of Child Health and Human Development Neonatal Research Network. Pediatrics. 2006 Jan; 117(1):84-92.
10. Faix RG, Kovarik SM, Shaw TR, Johnson RV. Mucocutaneous and invasive candidiasis among very low birth weight (less than 1,500 grams) infants in intensive care nurseries: a prospective study. Pediatrics. 1989 Jan;83(1):101-107

11. When to suspect fungal infection in neonates: A clinical comparison of Candida albicans and Candida parapsilosis fungemia with coagulase-negative staphylococcal bacteremia. Benjamin DK Jr, Ross K, McKinney RE Jr, Benjamin DK, Auten R, Fisher RG Pediatrics. 2000 Oct; 106(4):712-8.

12. Invasive Candida infections in the neonate. Brian Smith P, Steinbach WJ, Benjamin DK Jr Drug Resist Updat. 2005 Jun; 8(3):147-62.

13. Guidelines for treatment of candidiasis. Pappas PG, Rex JH, Sobel JD, Filler SG, Dismukes WE, Walsh TJ, Edwards JE, Infectious Diseases Society of America. Clin Infect Dis. 2004 Jan 15; 38(2):16189.

14. Pharmacokinetics and tissue penetration of fluconazole in humans.Brammer KW, Farrow PR, Faulkner JKRev Infect Dis. 1990 Mar-Apr; 12 Suppl 3():S318-26.

15. Manzoni P, Mostert M, Castagnola E Update on the management of Candida infections in preterm neonates Archives of Disease in Childhood - Fetal and Neonatal Edition 2015;100:F454-F459. 